Supplementary Material on

\title{
Time-Resolved Observation of Evolution of Amyloid- $\beta$ Oligomer with Temporary Salt Crystals
}

Kichitaro Nakajima ${ }^{1}$, Tomoya Yamazaki ${ }^{2}$, Yuki Kimura ${ }^{2}$, Masatomo $\mathrm{So}^{3}$, Yuji Goto ${ }^{3}$, and Hirotsugu $\mathrm{Ogi}^{{ }^{*}}$

1: Global Center for Medical Engineering and Informatics, Osaka University, Suita, Osaka 565-0871, Japan

2: Institute of Low Temperature Science, Hokkaido University, Sapporo 060-8638, Japan

3: Institute of Protein Research, Osaka University, Suita, Osaka 565-0871, Japan

4: Graduate School of Engineering, Osaka University, Suita, Osaka 565-0871, Japan

e-mail:ogi@prec.eng.osaka-u.ac.jp

\section{Supplementary Materials and Methods}

Procedure of the liquid-state transmission-electron-microscopy (LS-TEM) observation

First, a liquid cell chip (Poseidon Select, Protochips, USA) was cleaned by an oxygen plasma to make its surface hydrophilic. Second, the $A \beta$ sample solution was prepared as described in the main manuscript. After addition of a sample solution of $0.5 \mu \mathrm{L}$, the chip was carefully sealed to prevent the evaporation of the sample solution in a vacuum chamber. Because the actual volume in the chip is $\sim 1 \mathrm{~nL}$, a small amount of the sample solution remained inside the chip, and the other solution was removed from the chip part when the chip is sealed. Consequently, $\mathrm{A} \beta$ monomers of the order of $10^{9}$ were included in the chip. (We did not use a flow system in our experiments.) The chip was then inserted into the vacuum chamber of the TEM system by setting it on the TEM holder. The time between the solution preparation and the beginning of the TEM observation was typically 1 hour.

For the observation, we set the energy density of the electron beam to be less than 1,000 electrons $/ \mathrm{nm}^{2} \mathrm{~s}$. The electron-beam irradiation was performed with an intermission to suppress the effect of electron dosage. (Typically, we performed the 10-min irradiation for 1 hour.) During the electron-beam irradiation, the electron-beam spot was moved and the irradiation was interrupted frequently to minimize the irradiation effect; when we see something be noted, we monitored its dynamic behavior for a while. By this procedure, the observation was performed for approximately 30 hours. 


\section{Supplementary Figures S1-S7}

(a)

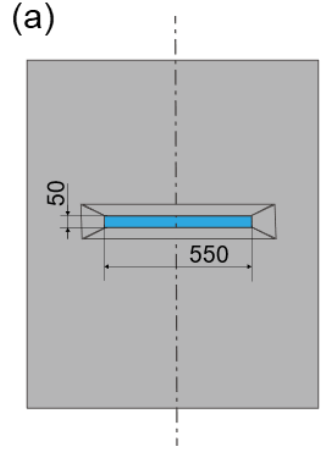

(c) (b)

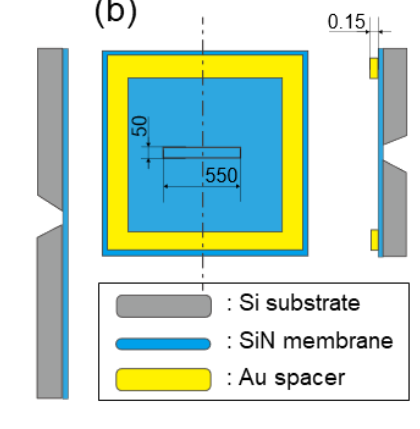

electron beam

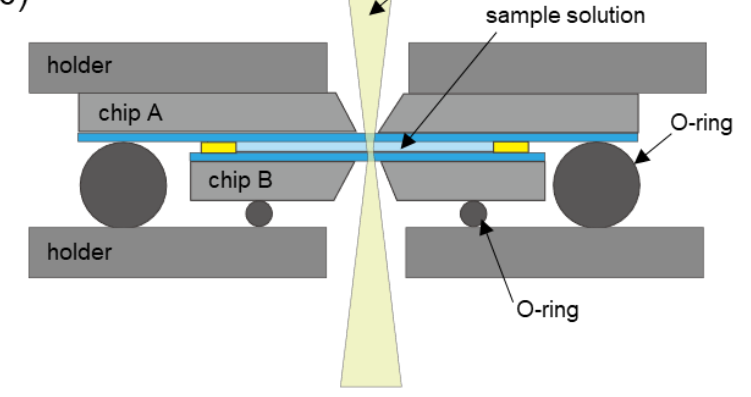

Fig. S1. Schematic illustrations of the liquid-state TEM observation. (a) Illustrations of chip A(EPT55W, Protochip, USA), and (b) chip B (EPB-55DS, Protochips, USA) (Dimensions shown are in micrometers). The left illustrations show the top views, and the right ones show the sectional views along the dashed lines. Thickness of the SiN membrane was $50 \mathrm{~nm}$, which allows transmission of the electron beam with low attenuation. Height of the Au spacer of chip B was $150 \mathrm{~nm}$, corresponding to the thickness of the sample solution. (c) A sectional illustration of the liquid-state TEM observation system. 

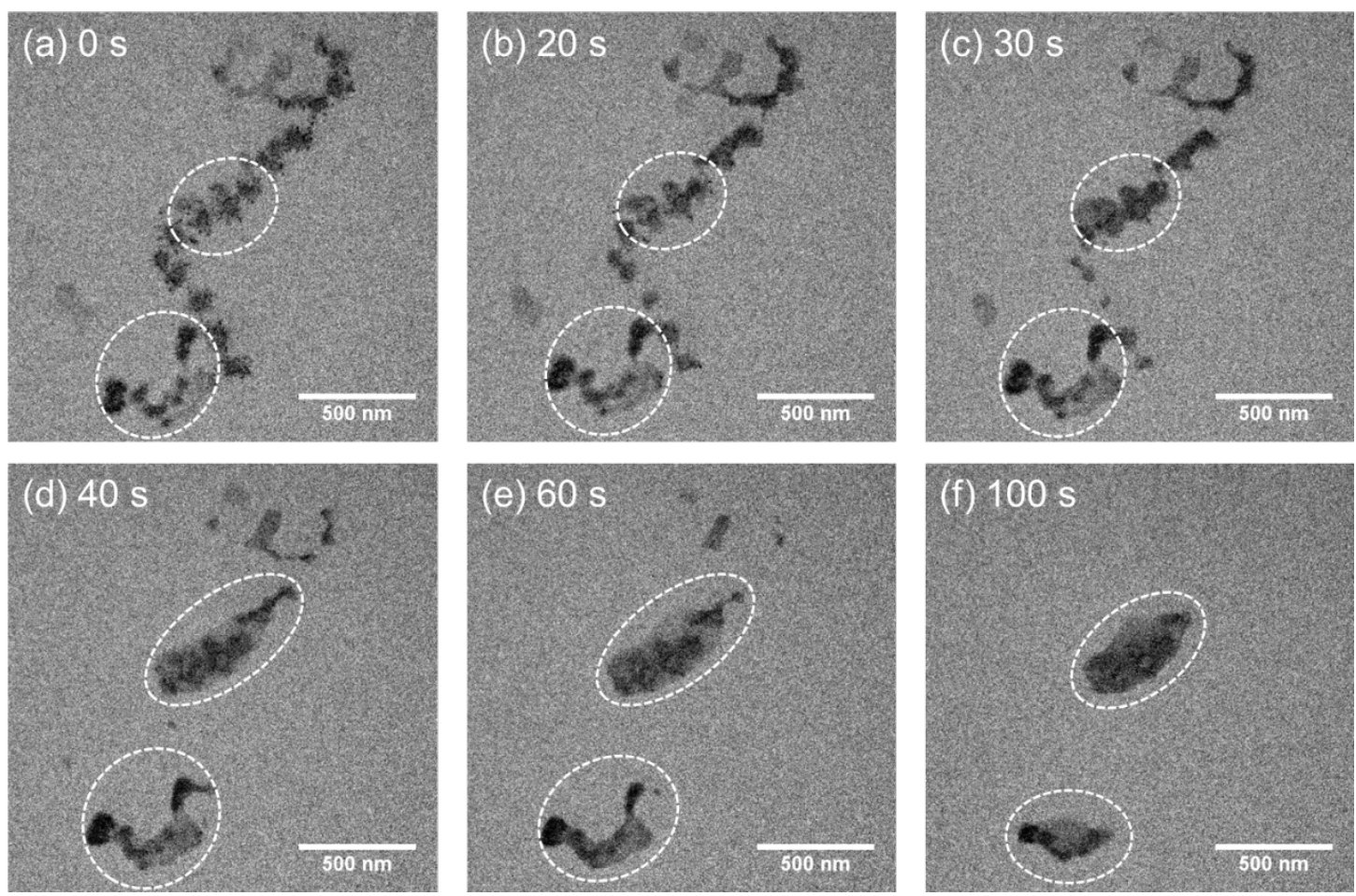

Fig. S2. Sequential snapshots during the morphology transformation of $A \beta_{1-40}$ aggregates from Fig. 1(d) into Fig. 1(e). The corresponding movie is Movie S1. White dashed circles indicate the stable aggregates used for the analysis in Fig. 1(f). They are defined as the aggregates remaining at $100 \mathrm{~s}$ in Movie S1. 

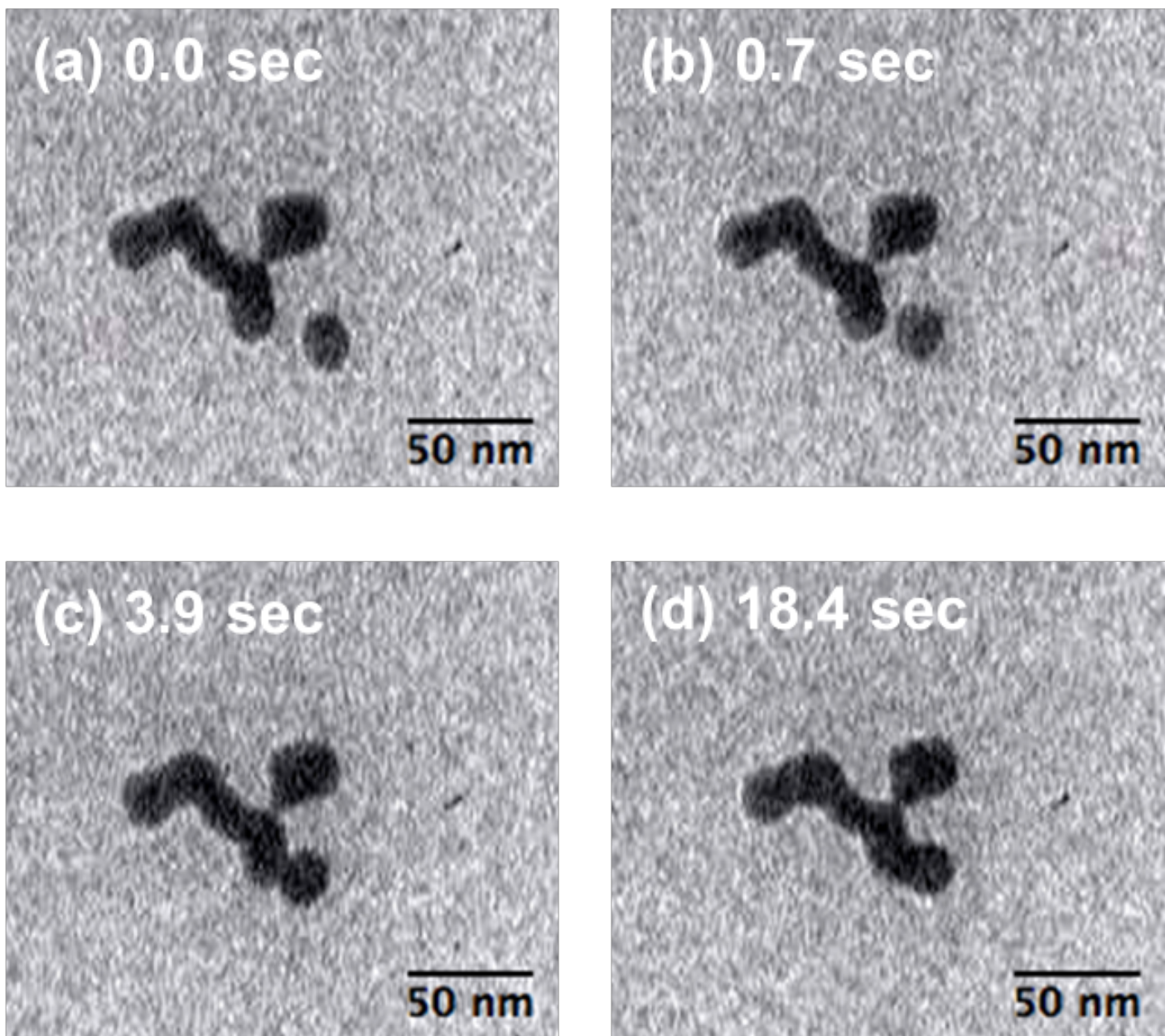

Fig. S3. Sequential snapshots of the coalescence event of the $A \beta_{1-40}$ spherical amorphous aggregates.

The corresponding movie is Movie S2. 

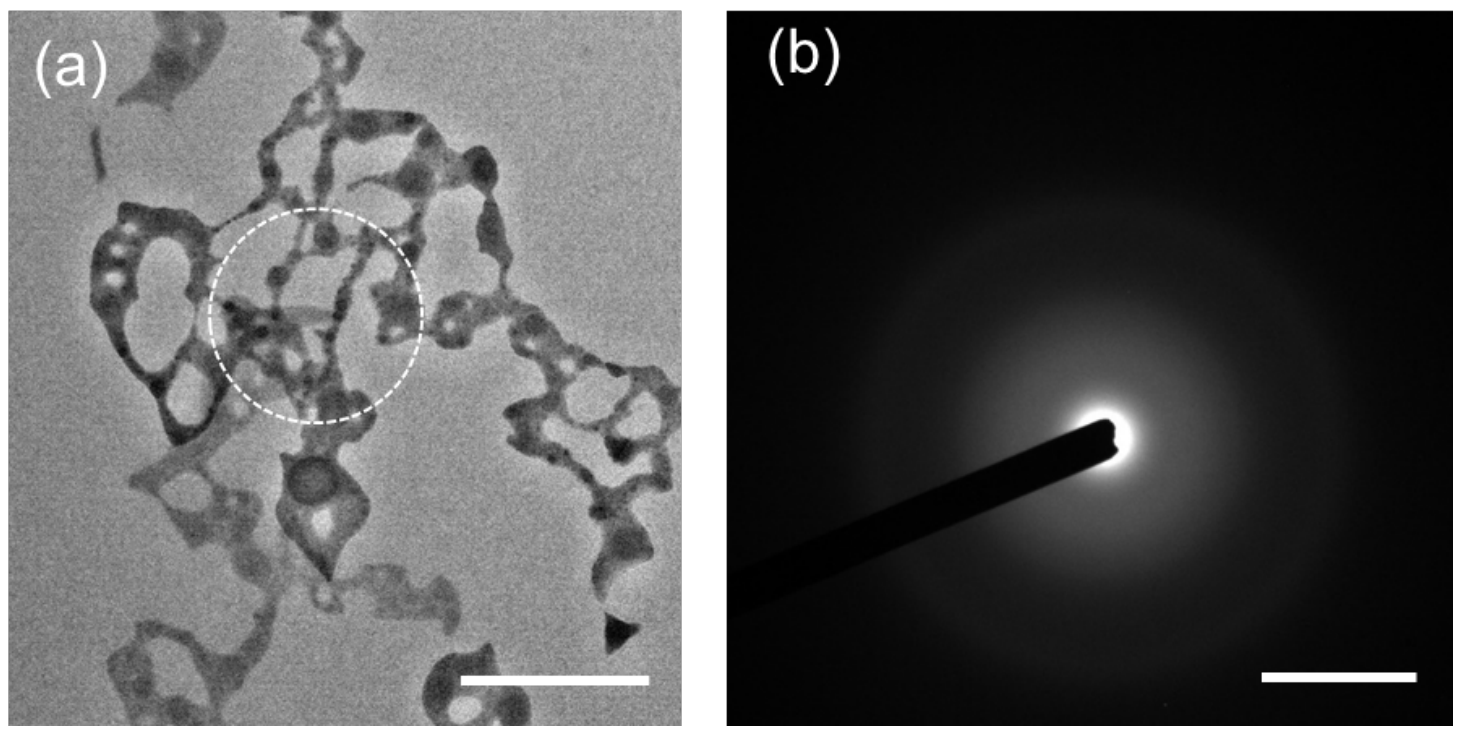

Fig. S4. Structural analysis of the network-like aggregate. (a) A TEM image of the network-like aggregate and (b) corresponding ED pattern. The scale bars in (a) and (b) are $200 \mathrm{~nm}$ and $5 \mathrm{~nm}^{-1}$, respectively. 


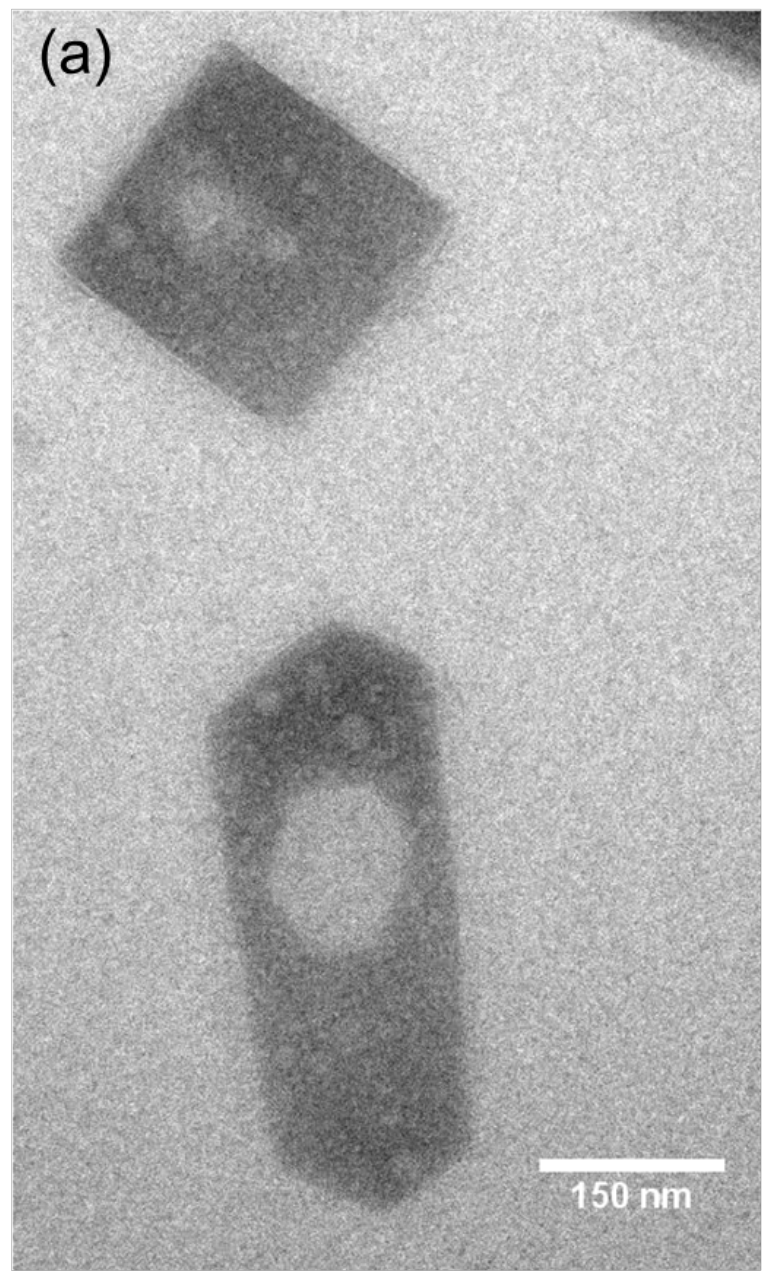

Fig. S5. Salt crystals observed in the present study. (b) A TEM image of rectangle-shape salt crystal, and (c) its ED pattern. The numbers in parentheses indicated by white arrows denote the corresponding diffraction planes of $\mathrm{NaCl}$.
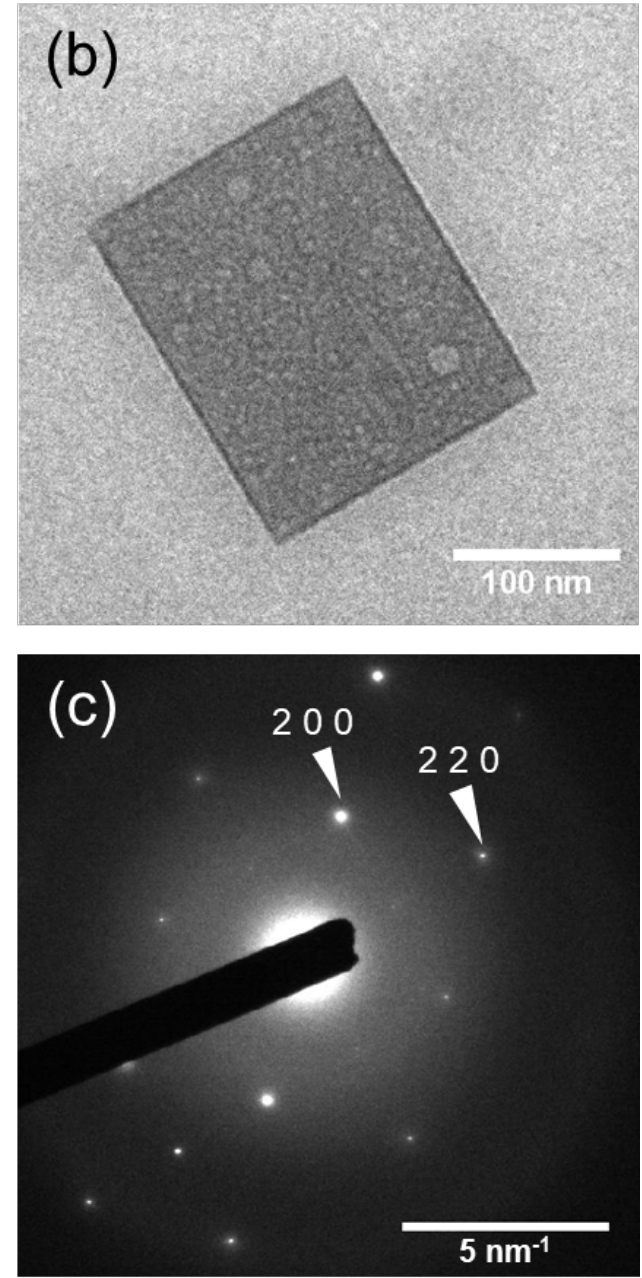

(a) TEM images of two kinds of salt crystals.

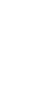



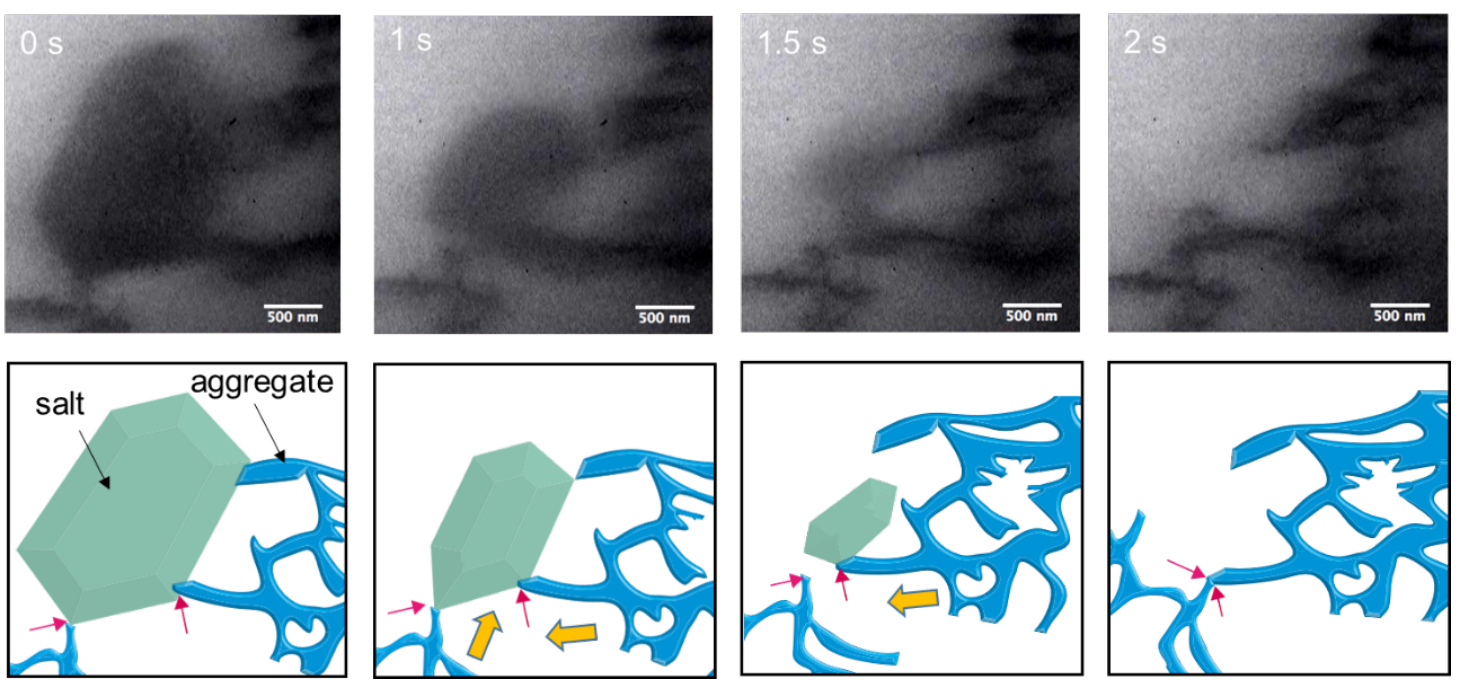

Fig. S6. Promotion of the binding reaction between the network-like aggregates by the salt-crystal dissolution. Terminals of two network-like aggregates are attached to the salt-crystal surface. Then, the terminals are dragged into the vanishing point of the salt crystal through the dissolution event, leading their binding reaction. Corresponding schematics are also shown. The corresponding movie is Movie S9. 

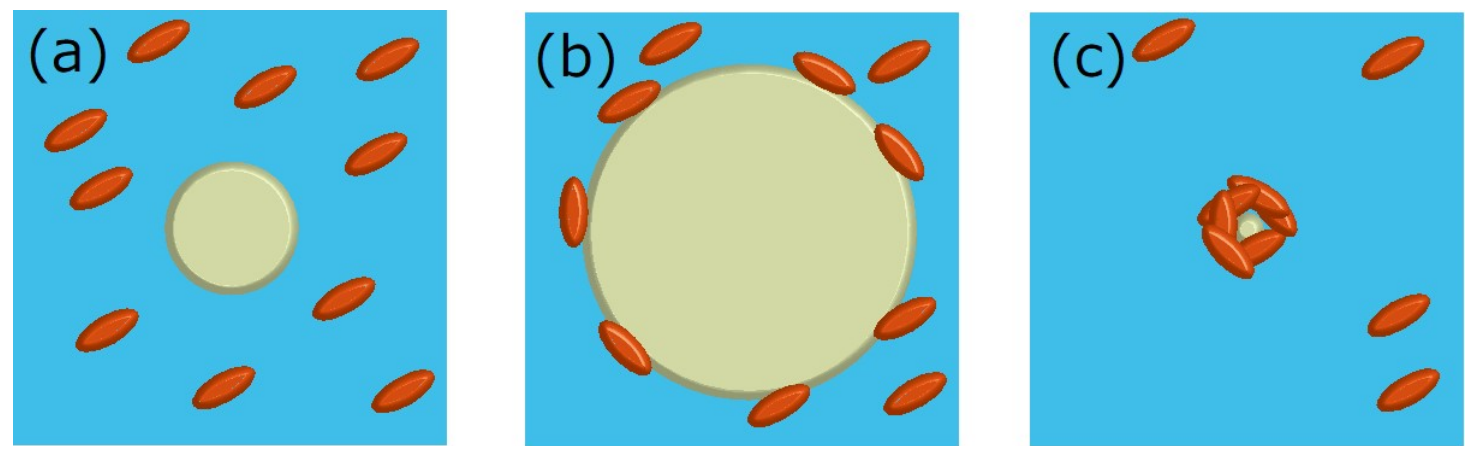

Fig. S7. Mechanism of aggregation acceleration by ultrasonic cavitation which is the dynamic gasliquid interface. (a) A cavitation bubble is generated by negative pressure of ultrasonic wave in solution, where protein molecules are dispersed. (b) The bubble radius increases during the negative pressure phase of the applied ultrasonic wave. In the growth phase, the protein molecules are attracted to the bubble surface through the hydrophobic interaction. (c) Finally, the bubble collapses by the positive pressure of the ultrasonic wave, inducing condensation of the protein molecules attracted to the bubble surface. 